\title{
A comparison of lumboperitoneal and ventriculoperitoneal shunting for idiopathic intracranial hypertension: an analysis of economic impact and complications using the Nationwide Inpatient Sample
}

\author{
Richard P. Menger, M.D., ${ }^{1}$ David E. Connor Jr., D.O., ${ }^{1}$ Jai Deep Thakur, M.D., ${ }^{1}$ \\ Ashish Sonig, M.D., ${ }^{1}$ Elainea Smith, B.A., ${ }^{2}$ Bharat Guthikonda, M.D., ${ }^{1}$ \\ ANd ANIL NANDA, M.D., M.P.H. ${ }^{1}$
}

${ }^{1}$ Department of Neurosurgery, Louisiana State University of Health Sciences; and ${ }^{2}$ Louisiana State University Health Shreveport School of Medicine, Shreveport, Louisiana

Object. Complications following lumboperitoneal (LP) shunting have been reported in $18 \%$ to $85 \%$ of cases. The need for multiple revision surgeries, development of iatrogenic Chiari malformation, and frequent wound complications have prompted many to abandon this procedure altogether for the treatment of idiopathic benign intracranial hypertension (pseudotumor cerebri), in favor of ventriculoperitoneal (VP) shunting. A direct comparison of the complication rates and health care charges between first-choice LP versus VP shunting is presented.

Methods. The Nationwide Inpatient Sample database was queried for all patients with the diagnosis of benign intracranial hypertension (International Classification of Diseases, Ninth Revision, code 348.2) from 2005 to 2009. These data were stratified by operative intervention, with demographic and hospitalization charge data generated for each.

Results. A weighted sample of 4480 patients was identified as having the diagnosis of idiopathic intracranial hypertension (IIH), with 2505 undergoing first-time VP shunt placement and 1754 undergoing initial LP shunt placement. Revision surgery occurred in $3.9 \%$ of admissions $(\mathrm{n}=98)$ for VP shunts and in $7.0 \%$ of admissions $(\mathrm{n}=123)$ for LP shunts $(\mathrm{p}<0.0001)$. Ventriculoperitoneal shunts were placed at teaching institutions in $83.8 \%$ of cases, compared with only $77.3 \%$ of first-time LP shunts $(\mathrm{p}<0.0001)$. Mean hospital length of stay (LOS) significantly differed between primary VP (3 days) and primary LP shunt procedures (4 days, $\mathrm{p}<0.0001)$. The summed charges for the revisions of $92 \mathrm{VP}$ shunts $(\$ 3,453,956)$ and those of the $6 \mathrm{VP}$ shunt removals $(\$ 272,484)$ totaled $\$ 3,726,352$ over 5 years for the study population. The summed charges for revision of 70 LP shunts $(\$ 2,229,430)$ and those of the $53 \mathrm{LP}$ shunt removals $(\$ 3,125,569)$ totaled $\$ 5,408,679$ over 5 years for the study population.

Conclusions. The presented results appear to call into question the selection of LP shunt placement as primary treatment for $\mathrm{IIH}$, as this procedure is associated with a significantly greater likelihood of need for shunt revision, increased LOS, and greater overall charges to the health care system.

(http://thejns.org/doi/abs/10.3171/2014.8.FOCUS14436)

\section{KeY WordS - intracranial hypertension - ventriculoperitoneal shunt • lumboperitoneal shunt $\quad$ health care charges $\quad$ socioeconomic outcomes}

$\mathrm{I}$ $\mathrm{N}$ patients with idiopathic intracranial hypertension (IIH) who are managed surgically, the default treatment has historically been placement of a lumboperitoneal (LP) shunt. Investigation is warranted to determine the demographics, outcomes, and health care charges of LP shunts as compared with traditional ventriculoperitoneal (VP) shunting. Despite the lack of prospective randomized studies, LP shunting is historically the first-line CSF diversion procedure for patients with IIH. ${ }^{28}$ The incidence

\footnotetext{
Abbreviations used in this paper: $\mathrm{CI}=$ confidence interval; ICD-9 = International Classification of Diseases, Ninth Revision; $\mathrm{IIH}=$ idiopathic intracranial hypertension; LOS = length of stay; LP = lumboperitoneal; NIS = Nationwide Inpatient Sample; OR = odds ratio; $\mathrm{VP}=$ ventriculoperitoneal.
}

of IIH ranges from 1 to 3 patients per 100,000 people per year. ${ }^{22}$ The annual cost of treating IIH in the US has been cited as $\$ 444$ million. ${ }^{9}$ A previous study by the senior author (A.N.) noted the overall cost of implanted ventricular shunts to be a $\$ 1$ billion problem..$^{19}$ The delivery of health care in the new marketplace will focus on patient outcomes as well as economic impact. Neurosurgeons will be forced to illustrate that interventions directly help the patient in an economically responsible fashion. Neurosurgeons must remain in the forefront of surgical interventions and must do so in a patient-centered but economically viable way. This study specifically uses the Nationwide Inpatient Sample (NIS) database to illustrate morbidity and the national estimates of health care charges related to LP versus VP shunting, specifically for IIH. 


\section{Methods}

\section{Database Description}

The NIS, the largest publicly available database detailing all-payer US inpatient care, contains data from 5 to 8 million hospital stays from approximately 1000 hospitals participating in the Healthcare Cost and Utilization Project. Data collected by the Agency for Healthcare Research and Quality is intended to approximate a $20 \%$ stratified sample of US community hospitals. The Clinical Classifications Software coding system was developed by the Agency for Healthcare Research and Quality as a tool for clustering 14,000 diagnosis codes and 3900 procedure codes from The International Classification of Diseases, Ninth Revision (ICD-9), into a manageable number of clinically meaningful categories.

\section{Data Extraction}

Having previously compiled the NIS database for the years 2005 to 2009, inclusive of data from 25,669 hospitals, onto a single server loaded with statistical analysis software (IBM SPSS Statistics version 20, and JMP version 9), a query was initiated for all patients admitted under the diagnosis of benign intracranial hypertension (ICD-9 code 348.2). Categorical variables and comorbidities extracted included sex, age $>65$ years, death during hospitalization, obesity, incidence of wound infection, diagnosis of meningitis, hospital location (rural vs urban), hospital teaching status, nonroutine (not home) discharge, and total length of stay (LOS). Routine discharge by definition was considered the expected discharge home or to self care.

Entries in the NIS database can be associated with up to 15 separate ICD-9 or Clinical Classifications Software codes for primary procedure/diagnosis. The data set was subsequently sorted by the following primary codes: 0234 for initial placement of a VP shunt, 0242 for replacement of a VP shunt, 0243 for removal of a VP shunt, 0371 for initial placement of an LP shunt, 0397 for revision of an LP shunt, and 0398 for removal of an LP shunt. To identify those cases of primary LP or VP shunt placement occurring after the revision or complete removal of a catheter system of the opposing category, all secondary codes were queried and compared with the primary diagnosis indicated for that encounter. The incidence of comorbidity was then approximated by the incidence of the complication against the entire subgroup (LP or VP shunting).

Total inpatient charges (TOTCHG), an NIS-coded variable, was identified in each case and cumulative charges were calculated for individual procedure codes by summing individual values. Length of stay, similarly coded under "LOS_X" in the database, was compared for all categorical variables and procedure codes. Nonroutine discharge was identified by NIS code $>1$ in the category "DISPUNIFORM," with in-hospital death identified by "DIED." Charges were attributed to the shunting procedure when it was deemed the primary code. The NIS database does not allow for specific patient outcomes at the individual level. Revision rates and removal rates were extrapolated back to index procedures as representative of estimated revision and removal rates over the 4-year period.

\section{Statistical Analysis}

Demographic, patient, and hospital factors underwent univariate analysis utilizing the Pearson chi-square test, followed by multivariate binary logistic regression, generating probability values, odds ratios (ORs), and the 95\% confidence intervals (CIs). To investigate intergroup differences, nonparametric (Kruskal-Wallis) testing was used to determine statistical significance, with a $\mathrm{p}$ value $<0.05$ considered significant.

\section{Results}

Four thousand four hundred eighty patients, identified as having the diagnosis of IIH according to the corresponding ICD-9 code, underwent CSF diversion from 2005 to 2009 . Two thousand five hundred five patients underwent first-time VP shunting, whereas 1754 underwent first-time LP shunting. Statistical trends are listed in Table 1. Data regarding sex was unavailable for 25 patients in the primary VP shunt group and 19 patients in the primary LP shunt group. Two hundred thirty-seven (9.6\%) of 2505 patients with VP shunts were male whereas $2243(90.4 \%)$ were female. One hundred ninety-five patients $(11.2 \%)$ with LP shunts were male, whereas 1540 (88.8\%) were female. The difference between groups was not statistically significant but did show a trend $(p=0.09)$. Age did not show a significant difference $(\mathrm{p}=0.17)$ between the VP and LP shunt groups with 34/2505 (1.4\%) and 15/1754 $(0.9 \%)$ greater than 65 years of age, respectively. The rate of meningitis was similar between the two groups as well $(\mathrm{p}=0.49) ; 20(0.8 \%)$ of 2505 patients with VP shunts developed meningitis compared with $10(0.6 \%)$ of 1754 patients with LP shunts. There were no wound infections in either series. Seven hundred thirty-six patients (29.4\%) with VP shunts were obese, compared with 480 (27.4\%) of the patients with LP shunts, which was not statistically significant $(\mathrm{p}=0.15)$. In the VP shunt group, 93.5\% had a routine discharge compared with $94.8 \%$ (1663/1754) in the LP shunt group, which trended toward a significant difference $(\mathrm{p}=0.067)$.

A total of 5 patients $(0.1 \%)$ died during hospitalization, all of them in the LP shunt group $(5 / 1754,0.3 \%)$, which was a statistically significant difference between groups $(\mathrm{p}=0.01)$. VP shunts were inserted at teaching institutions in $83.8 \%$ of cases $(2100 / 2505)$, versus only $77.3 \%$ (1355/1754) first-time LP shunts ( $p<0.0001)$. Sixty-five $(2.6 \%)$ VP shunts were placed in a rural hospital, compared with $4(0.2 \%)$ of 1754 LP shunts, which was significantly different $(\mathrm{p}<0.0001)$. A description of each of these variables can be found in Table 2 .

Revision or removal surgery occurred in $3.9 \%$ of admissions (98/2505) for VP shunts and in $7.0 \%$ of admissions (123/1754) for LP shunts ( $\mathrm{p}<0.0001$; Table 2$)$. Mean hospital LOS also differed significantly between primary VP (3.4 days) and primary LP shunt procedures ( 4 days; $p<0.0001$ ). The LOS ranged from 0 to 45 days for patients with a primary VP shunt and 0 to 38 days for patients with a primary LP shunt. There were no statisti- 
Analysis of shunting in idiopathic intracranial hypertension

TABLE 1: Descriptive and outcome relationships in patients undergoing CSF diversion with IIH

\begin{tabular}{|c|c|c|c|c|c|}
\hline Variable & VP Shunt & LP Shunt & $\mathrm{p}$ Value & OR & $95 \% \mathrm{Cl}$ \\
\hline total no. of patients & 2505 & 1754 & & & \\
\hline females & $2243 / 2480(90.4 \%)$ & $1540 / 1735(88.8 \%)$ & 0.0852 & & \\
\hline age $>65$ yrs & $34(1.4 \%)$ & $15(0.9 \%)$ & 0.1719 & & \\
\hline meningitis & $20(0.8 \%)$ & $10(0.6 \%)$ & 0.4924 & & \\
\hline obesity & $736(29.4 \%)$ & $480(27.4 \%)$ & 0.15 & & \\
\hline routine discharge & $2341(93.5 \%)$ & $1663(94.8 \%)$ & 0.067 & & \\
\hline $\begin{array}{l}\text { need for revision } \\
\text { mean LOS (days) }\end{array}$ & $98(3.9 \%)$ & $123(7.0 \%)$ & $<0.0001$ & 0.6 & $0.4-0.7$ \\
\hline for primary procedure & 3.4 & 4.0 & $<0.0001$ & 0.6 & $0.3-0.8$ \\
\hline for revision & 3.8 & 3.9 & 0.89 & & \\
\hline for removal & 6.0 & 6.1 & 0.97 & & \\
\hline teaching hospital & $2100(83.8 \%)$ & $1355(77.3 \%)$ & $<0.0001$ & 1.6 & $1.3-1.8$ \\
\hline rural location & $65(2.6 \%)$ & $4(0.2 \%)$ & $<0.0001$ & 11.7 & $4.2-32.1$ \\
\hline
\end{tabular}

* Length of stay in the VP shunt group ranged from 0 to 45 days, versus 0-38 days in the LP shunt group.

cally significant differences in LOS for VP or LP shunt revisions or removals ( $\mathrm{p}=0.89$ and 0.97 , respectively).

Mean charge for placement of a single primary VP shunt was $\$ 37,708$, compared with $\$ 32,617$ for placement of a primary LP shunt. The average charge for VP shunt revision was $\$ 37,543$, and $\$ 45,414$ for VP shunt removal. The mean charge for LP shunt revision was $\$ 32,849$, and for LP shunt removal was $\$ 58,973$. The summed charges for revision of $92 \mathrm{VP}$ shunts $(\$ 3,453,956)$ and for 6 VP shunt removals $(\$ 272,484)$ totaled $\$ 3,726,352$ over 5

TABLE 2: Description of variables and outcomes in patients undergoing primary and secondary CSF diversion in IIH

\begin{tabular}{|c|c|c|c|c|c|c|}
\hline \multirow[b]{2}{*}{ Characteristic } & \multicolumn{3}{|c|}{ VP Shunt } & \multicolumn{3}{|c|}{ LP Shunt } \\
\hline & Primary & Revision & Removal & Primary & Revision & Removal \\
\hline no. of cases & 2505 & 92 & 6 & 1754 & 70 & 53 \\
\hline \multicolumn{7}{|l|}{ death during hospitalization } \\
\hline no & 2505 & 92 & 6 & 1749 & 70 & 53 \\
\hline yes & 0 & 0 & 0 & 5 & 0 & 0 \\
\hline \multicolumn{7}{|l|}{ sex } \\
\hline male & 237 & 9 & 0 & 195 & 5 & 9 \\
\hline female & 2243 & 83 & 6 & 1540 & 65 & 44 \\
\hline \multicolumn{7}{|l|}{ obesity } \\
\hline no & 1769 & 67 & 0 & 1274 & 55 & 49 \\
\hline yes & 736 & 25 & 6 & 480 & 15 & 4 \\
\hline \multicolumn{7}{|l|}{ elderly (yrs) } \\
\hline$<65$ & 2471 & 92 & 6 & 1739 & 70 & 53 \\
\hline$>65$ & 34 & 0 & 0 & 15 & 0 & 0 \\
\hline \multicolumn{7}{|l|}{ meningitis } \\
\hline no & 2485 & 92 & 6 & 1744 & 70 & 53 \\
\hline yes & 20 & 0 & 0 & 10 & 0 & 0 \\
\hline \multicolumn{7}{|l|}{ wound infection } \\
\hline no & 2505 & 92 & 6 & 1754 & 70 & 53 \\
\hline yes & 0 & 0 & 0 & 0 & 0 & 0 \\
\hline \multicolumn{7}{|l|}{ hospital location } \\
\hline rural & 65 & 11 & 0 & 4 & 5 & 5 \\
\hline urban & 2440 & 81 & 6 & 1750 & 65 & 48 \\
\hline \multicolumn{7}{|l|}{ hospital teaching status } \\
\hline nonteaching & 405 & 34 & 0 & 399 & 22 & 30 \\
\hline teaching & 2100 & 58 & 6 & 1355 & 48 & 23 \\
\hline
\end{tabular}


years for the study population. The summed charges for revision of $70 \mathrm{LP}$ shunts $(\$ 2,229,430)$ and those for 53 LP shunt removals $(\$ 3,125,569)$ totaled $\$ 5,408,679$ over 5 years for the study population. The mean charge for patients with a complication of meningitis in the VP group was $\$ 69,820$, and $\$ 32,995$ for the LP shunt cohort. Charge analysis data can be found in Table 3 .

\section{Discussion}

The reality is that the treatment of IIH by CSF diversion is evolving, with both patient outcomes and economic implications as driving forces for change. In IIH, VP shunt placement has been proven as superior to LP shunting with clinical outcomes related to LOS, death, and revision surgery. Our study proves an economic benefit as well.

\section{Disease Process and Treatment}

Pseudotumor cerebri (or IIH) is a substantial economic problem that will only grow in the future. It is typically found in young women of a reproductive age and is linked to obesity. ${ }^{3}$ There has been documented resolution of symptoms after gastric banding procedures. ${ }^{24}$ From 1988 to 2002, the incidence of CSF shunting for IIH increased 350\%. ${ }^{6}$ The CSF shunting rate has increased at the same relative rate as morbid obesity. ${ }^{6}$ It is estimated that by 2030 all 50 states will have obesity rates of at least $44 \%$, and at least half of the population will be obese in 39 states. $^{23}$

CSF drainage is needed in the minority of patients with IIH who undergo unsuccessful conservative management. ${ }^{8}$ CSF drainage is an effective tool in treating $\mathrm{IIH},{ }^{1}$ as $95 \%$ of the patients demonstrate headache improvement in the immediate postoperative period. ${ }^{17}$ Eighty-three percent of patients undergoing VP shunt placement with the use of electromagnetic stereotactic image guidance experienced headache improvement at 3 years. ${ }^{12}$ Improved visual outcomes were also noted in a meta-analysis of VP shunt (38.7\%) and LP shunt (44.6\%) placement, but these rates were lower than those after optic nerve sheath decompression $(80 \%)^{?}$

TABLE 3: Charge analysis in patients undergoing CSF diversion for IIH

\begin{tabular}{lrcr}
\hline \multicolumn{1}{c}{ Procedure } & $\begin{array}{c}\text { No. of } \\
\text { Procedures }\end{array}$ & $\begin{array}{c}\text { Mean Charges } \\
\text { per Procedure }(\$)\end{array}$ & $\begin{array}{c}\text { Summed } \\
\text { Charges }(\$)\end{array}$ \\
\hline VP shunt placement & & & \\
$\quad$ primary & 2505 & 37,708 & $94,458,540$ \\
revision & 92 & 37,543 & $3,453,956$ \\
removal & 6 & 45,414 & 272,484 \\
revision or removal & 98 & 38,024 & $3,726,352$ \\
LP shunt placement & & & \\
primary & 1754 & 32,617 & $57,210,218$ \\
revision & 70 & 32,849 & $2,229,430$ \\
removal & 53 & 58,973 & $3,125,569$ \\
revision or removal & 123 & 43,973 & $5,408,679$ \\
\hline
\end{tabular}

The critical morbidity associated with shunting is the need for revision or additional surgery. Complication rates for additional surgery can range as high as $85.7 \% .^{13}$ The rate of revision surgery secondary to shunt malfunction or infection in the literature ranges from $38 \%$ to $85.7 \%$, with an overall rate of $52 \% .^{18}$ The consensus is that CSF manipulation is a reasonable intervention for improving visual outcome and headaches. ${ }^{18}$

\section{Comparison of CSF Diversion}

Our patient series continues to suggest that VP shunting is superior to LP shunting in patients with IIH. Patients with VP shunts underwent fewer revisions. Revision surgery occurred in 3.9\% of admissions for VP shunts and in $7.0 \%$ of admissions for LP shunts ( $p<0.0001)$. Patients with VP shunts had shorter average LOSs in the hospital compared with LP shunts (3 days compared with 4 days; $\mathrm{p}<0.001)$. VP shunt placements were also more likely to be performed at teaching institutions compared with LP shunts $(\mathrm{p}<0.0001)$.

This finding was similar to a retrospective finding in a study by McGirt et al. in 2004. These investigators reviewed shunt data over a 30 -year period, illustrating lower rates of obstruction and revision in their VP shunt series as compared with an LP shunt series. ${ }^{17}$ According to their data, LP shunts were associated with a 3 -fold increase in the risk of obstruction and a 2.5-fold increased risk of revision when compared with VP shunts. Overall, CSF drainage was successful, with $95 \%$ of patients receiving symptomatic relief from headaches.

The effectiveness and safety of VP shunts in the presence of IIH has been greatly increased with the advent of image guidance. Typical and historical aversion to the placement of a VP shunt is secondary to the standard slittype ventricles observed with the disease. Image guidance has allowed the procedure to be performed safely with great accuracy, with some series achieving 100\% placement of a VP shunt using image guidance with only 1 pass of the catheter needed on each patient. ${ }^{26}$ Initially, image guidance did require the morbidity and risks associated with cranial pinning including local trauma, hemodynamic considerations, and stress hormone release..$^{10}$ However, with the development of next-generation image guidance, pinning is avoided, providing the benefits of guidance without the morbidity of cranial pinning. . $11,14^{2}$

Overall, VP shunting with image guidance has been shown to be superior to the freehand technique. Concern still resides with surgeon experience and operative time. Using rigid cranial fixation image guidance, Sampath et al. illustrated that by using VP shunting with image guidance, time in the operative room was significantly increased but actual operative time was similar to published series using the freehand technique. ${ }^{21}$ All shunts were placed with ideal end points in 1 pass. The placement of VP shunts with image guidance shows increased accuracy and decreased proximal failures. ${ }^{25}$ In 2013, Wilson et al. published a retrospective cohort study, in which $12 \%$ of shunts placed by freehand for hydrocephalus, for all causes, developed a proximal shunt failure..$^{25}$ Overall there was a $16 \%$ failure rate with freehand shunt placement in this study. Only $4 \%$ of the patients undergoing 


\section{Analysis of shunting in idiopathic intracranial hypertension}

VP shunt placement with stereotactic guidance suffered a proximal failure, with a $10 \%$ overall failure rate. Increased importance of specific proximal placement is needed in the IIH patient subset. Given the small size of the ventricles, image guidance can be used to ensure good positioning and decrease the risk of proximal failure, improving the overall outcome of patients with IIH undergoing VP shunting.

In our series, there were significantly more LP shunt removals, which is likely multifactorial. The advent of adjustable VP shunt valves allows for a clinical period of change and intervention without full removal of the shunt catheter; ${ }^{27}$ this technology is generally not available in LP shunting. In fact, Lee et al. in 2014 noted significantly lower rates of VP shunt revision in patients with programmable shunts placed after subarachnoid hemorrhage. In evaluating 94 patients, $7 \%$ of patients with programmable devices underwent VP shunt revision, whereas $21 \%$ of patients with nonprogrammable shunts underwent revision between 2006 and 2013. ${ }^{16}$ Although complex, there were statistically significant decreases in overall complications, revision rates, overdrainage rates, and underdrainage rates in patients with VP programmable valves compared with nonprogrammable VP valves for shunting of all causes. ${ }^{27}$ In IIH, it is also possible that LP shunts represent a subset of patients considered to be marginal cases of IIH, whereas VP shunts, given their cranial nature, represent more definitive cases. Clinical information regarding patients and VP shunt selection type is not available in the NIS database.

Five deaths occurred in the LP shunt group, an unexpected outcome given the generally young population. Exact data extraction regarding each individual patient is unavailable given the constraints of the database. It can only be hypothesized that the generally overweight population is more predisposed to medical comorbidities associated with hospital stay and increased LOS, but we found no statistically significant difference in obesity rates between the VP and LP shunt cohorts. The 5 deaths also call into question iatrogenic hindbrain herniation. Case reports have indicated death from hindbrain herniation following LP shunting. ${ }^{5}$ In the pediatric population, as many as $70 \%$ of patients with LP shunts for all causes developed hindbrain herniation, ranging from $2 \mathrm{~mm}$ to $21 \mathrm{~mm},{ }^{4}$ but only $5 \%$ of the study population required intervention.

Furthermore, VP shunting has been determined to be economically effective in the treatment of IIH. The summed health care charges for revision of $92 \mathrm{VP}$ shunts over the 5-year period was $\$ 3,453,956$, and the summed charges of $6 \mathrm{VP}$ shunt removals was $\$ 272,484$, with the cumulative charges for additional surgery for VP shunts totaling $\$ 3,726,352$ over 5 years. The summed charges for revision of $70 \mathrm{LP}$ shunts over the 5 year period was $\$ 2,229,430$, and the summed charges of 53 LP shunt removals was $\$ 3,125,569$, with the cumulative charges for additional surgery for LP shunts totaling $\$ 5,408,679$ over 5 years.

Specifically, the average charge for LP shunt removal was $\$ 58,973$, while the average charge for VP shunt removal was $\$ 45,414$. LP shunts are more prone to migra- tion in failure presentation than VP shunts..$^{13}$ Karabatsou et al. in 2004 noted that $85.7 \%$ of patients with IIH and LP shunts needed shunt revision: 21 patients underwent 63 revisions, with 15 cases of abdominal migration, which in theory can be more complex and require a multidisciplinary approach. ${ }^{13}$ This fact, combined with the unique risk of iatrogenic hindbrain herniation at presentation requiring removal of LP shunts, likely plays a substantial role in the increased charges associated with LP shunt removal. Indeed, for simple revision, the difference in charges was mitigated, with $\$ 37,543$ for VP shunt revision and $\$ 32,829$ for LP shunt revision.

\section{New Treatment Considerations}

New treatment algorithms have developed for intervention in IIH involving the placement of transverse sinus stents. This modality has been proven to improve intracranial pressure in patients with a specific venogenic cause to IIH development. Within the literature, focal venous stenosis is present among 30\%-93\% of patients with IIH. ${ }^{15}$ Patients are considered candidates for stent placement with focal stenosis typically presenting in the transverse or sigmoid sinus. ${ }^{20}$ This procedure is technically feasible and highly effective, with a review noting improvement in headaches of as much as $88 \%$ and resolution of papilledema in $97 \%$ of patients. ${ }^{20}$ Complication rates were noted to be $6 \%$, including a $2 \%$ incidence of subdural hematoma. ${ }^{20}$ This new treatment modality looks to be a valuable tool in the specific subset of patients with IIH and focal venous stenosis.

In a cost analysis by Ahmed et al., this modality was deemed cost neutral to shunt placement, with a mean cost of $\$ 15,797$ for an initial CSF shunt and $\$ 13,863$ for an initial stenting procedure. However, shunting had a statistically significantly higher rate of failure requiring an additional procedure. Over a 12-year period, only $13 \%$ of the stenting group needed additional stent procedures, whereas $55 \%$ of the shunting group required an additional shunt procedure. ${ }^{2}$ This, however, does not fully take into account the added superiority of image guidance techniques now available.

\section{Limitations of the Study}

It is important to recognize that our analysis represents a component of economic evaluation between the two shunting modalities and an estimate of revision and removal rates. The cost related to outpatient follow-up in the clinic, shunt reprogramming for VP shunts with adjustable valves, and imaging coordination with outpatient imaging centers is also not captured. This missing information represents a very real and tangible economic impact that may increase the cost of VP shunt placement (an increase in return visits for programmable valve manipulation).

In our review we were limited by information provided by retrospective review, the limitations found in the NIS database. The NIS database does not provide clinical information about specific cases of IIH. It lacks evaluation of long-term data of the posthospitalization course, follow-up, or procedures performed outside of hospitals 
participating in the database. No data are available about specific patients, types of shunting catheters, or time frame between revisions. This is an inherent limitation associated with the NIS database study, and therefore the purpose of the current study is to show the gross trends between the 2 groups in the treatment of IIH with pertinent possible subgroup analysis. Furthermore, the database does not allow one to follow a single patient, and the readmission rate of the same patient later, which may not be cumulative. Pertinent to the calculation of the failure rates, the study truly measures the prevalence of the failure rates rather than the true incidence. However, previous NIS database studies have suggested the validity of universal exploration of NIS databases with certain cautions. Likewise, clinical decision-making rubrics for shunting decisions were not made available and specific clinical indications or reasoning cannot be deciphered, other than ICD-9 coding. Specifically, it was not possible to stratify the use of image guidance in the placement of VP shunts.

\section{Future Considerations}

This study's results are applicable for demonstrating the superiority of VP shunting in terms of average LOS, revision, and charges. The results do not advocate for the unilateral decision to perform VP shunting over LP shunting in all scenarios. Clinical expertise, surgeon comfort, and availability of technology all help to formulate clinical decision making on a patient-by-patient basis. This study also did not evaluate and cannot be interpreted to show a benefit in relief of symptoms (headache, visual deficit) and quality of life improvements between the two study arms.

Investigation moving forward must focus on the clinical decision-making process as well as patient- and provider-specific demographics regarding the decision to place VP shunts as compared with LP shunts. Careful patient selection and subgroup analysis may be needed to develop guidelines. Information from this study questions the historical practice of first-choice LP shunting for IIH and lays the foundation for future investigation with randomized controlled trial evaluation to determine the most economically and outcome-favorable decision model for CSF diversion in patients with IIH.

\section{Conclusions}

In our patient series, VP shunts have been proven to be superior to LP shunts secondary to IIH in terms of revision surgery and average hospital LOS. ${ }^{17}$ The proliferation of image guidance technology allows accurate VP shunt placement in slit ventricle syndromes. ${ }^{21,26}$ Our study directly adds an economic benefit and advantage to the primary placement of VP shunts.

\section{Disclosure}

The authors report no conflict of interest concerning the materials or methods used in this study or the findings specified in this paper.

Author contributions to the study and manuscript preparation include the following. Conception and design: Menger, Connor,
Smith, Guthikonda, Nanda. Acquisition of data: Connor, Sonig. Analysis and interpretation of data: Menger, Connor, Thakur. Drafting the article: Menger, Connor. Critically revising the article: Menger, Connor, Thakur, Guthikonda. Reviewed submitted version of manuscript: Menger, Guthikonda. Statistical analysis: Connor, Sonig. Study supervision: Menger. Literature search: Smith, Nanda.

\section{References}

1. Abubaker K, Ali Z, Raza K, Bolger C, Rawluk D, O'Brien D: Idiopathic intracranial hypertension: lumboperitoneal shunts versus ventriculoperitoneal shunts - case series and literature review. Br J Neurosurg 25:94-99, 2011

2. Ahmed RM, Zmudzki F, Parker GD, Owler BK, Halmagyi GM: Transverse sinus stenting for pseudotumor cerebri: a cost comparison with CSF shunting. AJNR Am J Neuroradiol 35:952-958, 2014

3. Andrews LE, Liu GT, Ko MW: Idiopathic intracranial hypertension and obesity. Horm Res Paediatr 81:217-225, 2014

4. Chumas PD, Armstrong DC, Drake JM, Kulkarni AV, Hoffman HJ, Humphreys RP, et al: Tonsillar herniation: the rule rather than the exception after lumboperitoneal shunting in the pediatric population. J Neurosurg 78:568-573, 1993

5. Chumas PD, Drake JM, Del Bigio MR: Death from chronic tonsillar herniation in a patient with lumboperitoneal shunt and Crouzon's disease. Br J Neurosurg 6:595-599, 1992

6. Curry WT Jr, Butler WE, Barker FG II: Rapidly rising incidence of cerebrospinal fluid shunting procedures for idiopathic intracranial hypertension in the United States, 1988-2002. Neurosurgery 57:97-108, 2005

7. Feldon SE: Visual outcomes comparing surgical techniques for management of severe idiopathic intracranial hypertension. Neurosurg Focus 23(5):E6, 2007

8. Friedman DI, Jacobson DM: Idiopathic intracranial hypertension. J Neuroophthalmol 24:138-145, 2004

9. Friesner D, Rosenman R, Lobb BM, Tanne E: Idiopathic intracranial hypertension in the USA: the role of obesity in establishing prevalence and healthcare costs. Obes Rev 12:e372e380, 2011

10. Geze S, Yilmaz AA, Tuzuner F: The effect of scalp block and local infiltration on the haemodynamic and stress response to skull-pin placement for craniotomy. Eur J Anaesthesiol 26:298-303, 2009

11. Hayhurst C, Byrne P, Eldridge PR, Mallucci CL: Application of electromagnetic technology to neuronavigation: a revolution in image-guided neurosurgery. Technical note. J Neurosurg 111:1179-1184, 2009

12. Kandasamy J, Hayhurst C, Clark S, Jenkinson MD, Byrne P, Karabatsou K, et al: Electromagnetic stereotactic ventriculoperitoneal CSF shunting for idiopathic intracranial hypertension: a successful step forward? World Neurosurg 75:155160, 32-33, 2011

13. Karabatsou K, Quigley G, Buxton N, Foy P, Mallucci C: Lumboperitoneal shunts: are the complications acceptable? Acta Neurochir (Wien) 146:1193-1197, 2004

14. Kim YB, Lee JW, Lee KS, Lee KC: Image-guided placement of ventricular shunt catheter. J Clin Neurosci 13:50-54, 2006

15. Kumpe DA, Bennett JL, Seinfeld J, Pelak VS, Chawla A, Tierney M: Dural sinus stent placement for idiopathic intracranial hypertension. Clinical article. J Neurosurg 116:538-548, 2012

16. Lee L, King NKK, Kumar D, Ng YP, Rao J, Ng H, et al: Use of programmable versus nonprogrammable shunts in the management of hydrocephalus secondary to aneurysmal subarachnoid hemorrhage: a retrospective study with cost-benefit analysis. Clinical article. J Neurosurg 121:899-903, 2014

17. McGirt MJ, Woodworth G, Thomas G, Miller N, Williams M, Rigamonti D: Cerebrospinal fluid shunt placement for pseudotumor cerebri-associated intractable headache: predictors of 


\section{Analysis of shunting in idiopathic intracranial hypertension}

treatment response and an analysis of long-term outcomes. J Neurosurg 101:627-632, 2004

18. Mukherjee N, Bhatti MT: Update on the surgical management of idiopathic intracranial hypertension. Curr Neurol Neurosci Rep 14:438, 2014

19. Patwardhan RV, Nanda A: Implanted ventricular shunts in the United States: the billion-dollar-a-year cost of hydrocephalus treatment. Neurosurgery 56:139-145, 2005

20. Puffer RC, Mustafa W, Lanzino G: Venous sinus stenting for idiopathic intracranial hypertension: a review of the literature. J Neurointerv Surg 5:483-486, 2013

21. Sampath R, Wadhwa R, Tawfik T, Nanda A, Guthikonda B: Stereotactic placement of ventricular catheters: does it affect proximal malfunction rates? Stereotact Funct Neurosurg 90: 97-103, 2012

22. Tarnaris A, Toma AK, Watkins LD, Kitchen ND: Is there a difference in outcomes of patients with idiopathic intracranial hypertension with the choice of cerebrospinal fluid diversion site: a single centre experience. Clin Neurol Neurosurg 113:477-479, 2011

23. Voelker R: Escalating obesity rates pose health, budget threats. JAMA 308:1514, 2012

24. Williams A, Morgan J, Johnson A, Bates S, Pople I, Norton $\mathrm{S}$ : Resolution of pseudotumor cerebri following surgery for morbid obesity. J Surg Case Rep 2010:7, 2010

25. Wilson TJ, Stetler WR Jr, Al-Holou WN, Sullivan SE: Com- parison of the accuracy of ventricular catheter placement using freehand placement, ultrasonic guidance, and stereotactic neuronavigation. Clinical article. J Neurosurg 119:66-70, 2013

26. Woodworth GF, McGirt MJ, Elfert P, Sciubba DM, Rigamonti D: Frameless stereotactic ventricular shunt placement for idiopathic intracranial hypertension. Stereotact Funct Neurosurg 83:12-16, 2005

27. Xu H, Wang ZX, Liu F, Tan GW, Zhu HW, Chen DH: Programmable shunt valves for the treatment of hydrocephalus: a systematic review. Eur J Paediatr Neurol 17:454-461, 2013

28. Yadav YR, Parihar V, Agarwal M, Bhatele PR, Saxena N: Lumbar peritoneal shunt in idiopathic intracranial hypertension. Turk Neurosurg 22:21-26, 2012

Manuscript submitted July 14, 2014.

Accepted August 18, 2014.

Portions of this project were presented in abstract form as an oral poster at the 2013 CNS Annual Meeting in San Francisco, California, October 19-23, 2013.

Please include this information when citing this paper: DOI: 10.3171/2014.8.FOCUS14436.

Address correspondence to: Anil Nanda, M.D., M.P.H., LSU Health Sciences Center-Shreveport, Department of Neurosurgery, 1501 Kings Highway, P.O. Box 33932, Shreveport, LA 71130-3932. email: ananda@lsuhsc.edu. 